\title{
RADIANT HEATING OF LIQUID IN THERMAL ENTRY LENGHT
}

\author{
G. Medvedev ${ }^{1, *}$, and $A$. Martynova ${ }^{1}$ \\ ${ }^{1}$ National Research Tomsk Polytechnic University, 634050 Tomsk, Russia
}

\begin{abstract}
The given paper presents the calculation method of liquid flow and radiation heating in thermal entry length. For the solution of a problem with nonlinear boundary conditions the method of linear function is used [2] which allows to putting boundary conditions in a linear form.
\end{abstract}

\section{Problem statement}

Inside the thin-walled channel (fig.1) liquid fluid is moved and heated axially owing to outer high-temperature gas filling. The heat transfer on the outer surface keeps in both with the Stefan-Boltzmann's law. In the tube entry velocity traverse is parabolic, and the liquid temperature is constant and equal to $\theta_{0}$. Assume the viscosity factor is dependent on temperature and all other physical properties are permanent. It is reasonable that density is constant, weakly dependent on temperature for most of liquid fluids excluding from consideration influence of natural convection. The friction heat and axial heat transfer are neglected.

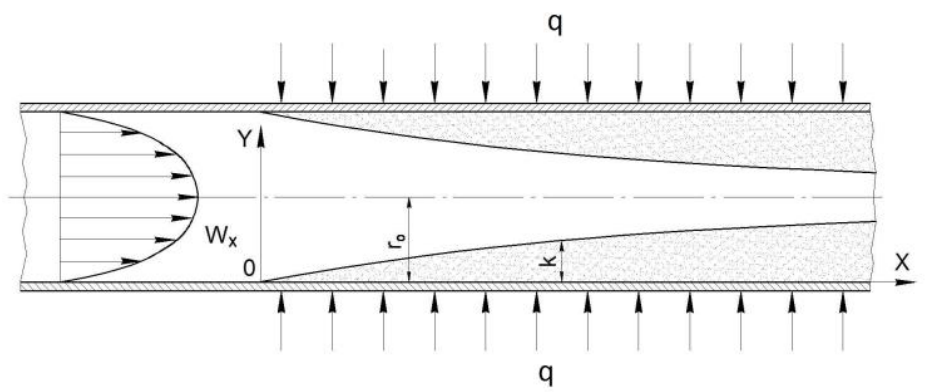

Fig.1. The physical model and coordinate system for thermal entry length.

Within specified conditions the heat transfer process is described by energy equation

\footnotetext{
*Corresponding author: ggm@tpu.ru
} 


$$
a \frac{1}{r} \cdot \frac{\partial}{\partial r}\left(r \frac{\partial \theta}{\partial r}\right)=W_{x} \frac{\partial \theta}{\partial x}+W_{r} \frac{\partial \theta}{\partial r}
$$

equation of through flow

$$
\frac{\partial W_{x}}{\partial x}+\frac{1}{r} \cdot \frac{\partial}{\partial r}\left(r \cdot W_{r}\right)=0
$$

and motion

$$
\frac{1}{r} \cdot \frac{\partial}{\partial r}\left(r \mu \frac{\partial W_{x}}{\partial r}\right)=\frac{\partial P}{\partial x}+\rho\left(W_{x} \frac{\partial W_{x}}{\partial x}+W_{r} \frac{\partial W_{x}}{\partial r}\right)-\frac{\partial}{\partial x}\left(\mu \frac{\partial W_{x}}{\partial x}\right)-\frac{\partial \mu}{\partial r} \cdot \frac{\partial W_{r}}{\partial x} .
$$

Here $\theta_{0}=\frac{T_{0}}{T_{c}} \leq \theta=\frac{T}{T_{c}}<1 ; T_{c}$ is temperature of heating medium, and $T_{0}$ is temperature of inlet flow.

The boundary conditions for the energy equation (1) reduce to inlet temperature

$$
\theta=\theta_{0} \text { with } x=0 \text { and } r=0
$$

to symmetry condition on pipe axis

$$
\frac{\partial \theta}{\partial r}=0, \text { with } x \geq 0 \text { and } r=0
$$

and to the law of radiant heating on surface

$$
\frac{\partial \theta}{\partial r}=\frac{\sigma T_{c}^{3}}{\lambda}\left(1-\theta^{4}\right) \frac{d}{d_{0}}, \text { with } x \geq 0 \text { and } r=r_{0} .
$$
$[2]$

To linearize the boundary conditions (6) we introduce nonlinear integral transformation

$$
Z=\int_{0}^{\theta} \frac{d \theta}{1-\theta^{4}}=\frac{1}{2}(\operatorname{arth} \theta+\operatorname{arctg} \theta)
$$

The transformation (7) linearizing the boundary condition (6)

$$
\frac{\partial Z}{\partial r}=\frac{\sigma \cdot T_{c}^{3} \cdot r_{0}}{\lambda} \cdot \frac{d}{d_{0}}, \text { with } x \geq 0 \text { and } r=r_{0},
$$


reduces the energy equation (1) to form:

$$
a \frac{1}{r} \cdot \frac{\partial}{\partial r}\left(r \frac{\partial Z}{\partial r}\right)-a F=W_{x} \frac{\partial Z}{\partial x}+W_{r} \frac{\partial Z}{\partial r}
$$

where

$$
F=\frac{4 \theta^{3}}{\left(1-\theta^{4}\right)^{2}} \cdot\left(\frac{\partial \theta}{\partial r}\right)^{2}
$$

In this connection the symmetry condition (5) isn't changed

$$
\frac{\partial Z}{\partial r}=0, \text { with } x \geq 0 \text { and } r=0 \text {, }
$$

and the transformed inlet temperature may be written as

$$
Z=\frac{1}{2}\left(\operatorname{arth} \theta_{0}+\operatorname{arctg} \theta_{0}\right)=Z_{0} \text { with } x \geq 0 \text { and } 0 \leq r \leq r_{0} .
$$

As is shown in [2-3] with not-too-large amounts of temperature gradients $\partial \theta / \partial Z$ the nonlinear complex (9) in the energy equation (8) may be neglected.

With further analysis we distinguish two zones in thermal entry length: thermal boundary layer with thickness $\Delta$ and liquid flow core, where temperature is constant and equal to inlet heated length temperature. It is considered that the amount $\Delta$ is substantially smaller than the pipe radius $r_{0}$. The last condition means that the considered problem is constrained by a set of small calculated lengths.

The using nondimensional variables

$$
\begin{gathered}
t=\frac{Z}{S k}, \quad S K=\frac{\sigma \cdot T_{c}^{3} \cdot r_{0}}{\lambda} \cdot \frac{d}{d_{0}}, \quad W_{x}=\frac{w_{x}}{w}, \quad W_{y}=\frac{w_{y}}{w}, \\
M=\frac{\mu}{\mu_{0}}, \quad P e=\frac{\bar{w} \cdot 2 r_{0}}{a}, \quad X=\frac{x}{r_{0}}, \quad Y=1-\frac{r}{r_{0}}, \quad \mathrm{~K}=\frac{\Delta}{r_{0}}
\end{gathered}
$$

and averaging right-hand sides of energy and motion equations relatively on thermal boundary layer thickness and pipe radius (like in [1]), we shall obtain 


$$
\begin{gathered}
\frac{1}{1-Y} \cdot \frac{\partial}{\partial Y}\left[(1-Y) \frac{\partial t}{\partial Y}\right]=B(X), \\
\frac{\partial W_{x}}{\partial X}+\frac{1}{1-Y} \cdot \frac{\partial}{\partial Y}\left[(1-Y) W_{y}\right]=0, \\
B(X)=\frac{P e}{2 k} \int_{0}^{k}\left(W_{x} \frac{1}{\partial X}+W_{y} \frac{\partial}{\partial Y}\right) d Y, \text { and } A(X) \text { is defined in [1]. }
\end{gathered}
$$

The solution of (10) and (12) must satisfy the boundary conditions

$$
\begin{array}{cl}
\frac{\partial t}{\partial Y}=-1, \quad W_{x}=W_{y}=0 \text { with } X \geq 0 \text { and } Y=0, \\
t_{0}=\frac{Z_{0}}{S k}, \frac{\partial t}{\partial Y}=0 & \text { with } X \geq 0 \text { and } 1 \geq Y \geq k, \\
\frac{\partial W_{x}}{\partial Y}=0 & \text { with } X \geq 0 \text { and } Y=1 .
\end{array}
$$

The integral transformed by means of the system (10) - (15) was outlined in the paper [1] and in general terms is expressed by dependence

$$
t=\frac{Z_{0}}{S k}+\frac{1}{k(2-k)}\left[-(1-k)^{2} \operatorname{Ln} \frac{1-Y}{1-k}-Y\left(1-\frac{Y}{2}\right)+k\left(1-\frac{k}{2}\right)\right]
$$

Inserting then $Z=S k \cdot t$ in relation (7) we define the target relative temperature $\theta$.

The expression for nondimensional temperature on the wall surface we can obtain from (16), assuming $Y=0$

$$
t_{\text {suf }}=\frac{Z_{0}}{S k}+\frac{1}{k(2-k)}\left[(1-k)^{2} \operatorname{Ln}(1-k)+k\left(1-\frac{k}{2}\right)\right]
$$

The most simple dependencies for temperature profile are obtained in the problems when liquid viscosity is constant. With $\mu=$ const the boundary layer thickness is defined from comparatively simple equation [17] 


$$
k^{3}\left(1-\frac{k}{5}\right)=24 \frac{x}{P e \cdot 2 r_{0}}
$$

\section{Results}

The fig. (2) shows temperature profiles in thermal entry length which were obtained for that conditions. The calculations are made on the basis of (18), (16) and (7). The inlet temperature $\theta_{0}$ is assumed equal to 0.5 . Nondimensional boundary layer thickness $k$ is chosen within ranges 0.10 and 0.20 . The calculated lengths $x / P e \cdot 2 r_{0}: 0.41 \cdot 10^{-4} ; 3.2 \cdot 10^{-4}$ match the chosen values of $k$.
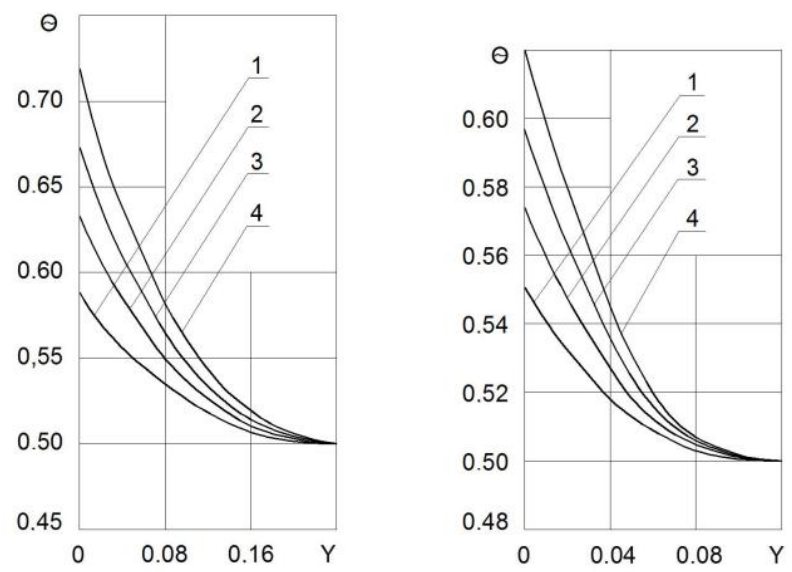

$$
k=0.2 ; \quad x / P e \cdot 2 r_{0}=3.2 \cdot 10^{-4}
$$$$
k=0.1 ; \quad x / P e \cdot 2 r_{0}=0.4 \cdot 10^{-4}
$$

Fig. 2. Temperature profiles in thermal entry length, $1-4: S k=1.0 ; 1.5 ; 2.0 ; 2.5$.

Here it may be noted that in conditions of constant viscosity the velocity profile is parabolic and it is possible to use exact solution of energy equation without averaging its righthand side.

\section{References}

1. B.S. Petukhov, Heat transfer and resistance within laminar flow in tubes (Energiya, Moscow, 1967) [in Russian]

2. V.V. Ivanov, G.G. Medvedev, Energy and transport 3 (1970) [in Russian]

3. V.V. Ivanov, The physics and chemistry of materials processing 5 (1972) [in Russian] 